\title{
3D kinematics through the X-shaped Milky Way bulge ${ }^{\star \star \star \star}$
}

\author{
S. Vásquez ${ }^{1,2,3}$, M. Zoccali ${ }^{1,3}$, V. Hill ${ }^{4}$, A. Renzini ${ }^{5,6}$, O. A. González ${ }^{2,3}$, E. Gardner $^{7}$, V. P. Debattista ${ }^{8}$, \\ A. C. Robin ${ }^{7}$, M. Rejkuba ${ }^{9}$, M. Baffico ${ }^{1}$, M. Monelli ${ }^{10,11}$, V. Motta ${ }^{12}$, and D. Minniti ${ }^{1,3,13}$ \\ ${ }^{1}$ Instituto de Astrofísica, Facultad de Física, Pontificia Universidad Católica de Chile, Av. Vicuña Mackenna 4860, Santiago, Chile \\ e-mail: [svasquez;mzoccali]@astro.puc.cl \\ ${ }^{2}$ European Southern Observatory, Alonso de Cordova 3107, Santiago, Chile \\ 3 The Milky Way Millennium Nucleus, Av. Vicuña Mackenna 4860, 782-0436 Macul, Santiago, Chile \\ ${ }^{4}$ Laboratoire Lagrange, UMR 7293, Université de Nice Sophia-Antipolis, CNRS, Observatoire de la Côte d'Azur, BP4229, \\ 06304 Nice, France \\ 5 INAF - Osservatorio Astronomico di Padova, vicolo dell'Osservatorio 5, 35122 Padova, Italy \\ 6 GEPI, Observatoire de Paris, CNRS, UMR 8111, Université Paris Diderot, 92125 Meudon, France \\ 7 Institut Utinam, CNRS UMR 6213, OSU THETA, Université de Franche-Comté, 41bis avenue de l'Observatoire, 25000 Besançon, \\ France \\ 8 Jeremiah Horrocks Insitute, University of Central Lancashire, Preston, PR1 2HE, UK \\ 9 European Southern Observatory, Karl-Schwarzschild-Strasse 2, 85748 Garching, Germany \\ 10 Instituto de Astrofísica de Canarias, Calle Vía Láctea s/n, 38205 La Laguna, Tenerife, Spain \\ 11 Departmento de Astrofísica, Universidad de La Laguna, 38200 La Laguna, Tenerife, Spain \\ 12 Departamento de Física y Astronomía, Universidad de Valparaiso, Avenida Gran Bretana 1111 Valparaiso, Chile \\ 13 Vatican Observatory, V00120 Vatican City State, Italy
}

Received 14 August 2012 / Accepted 23 April 2013

\begin{abstract}
Context. It has recently been discovered that the Galactic bulge is X-shaped, with the two southern arms of the X both crossing the lines of sight at $l=0$ and $|b|>4$, hence producing a double red clump in the bulge color magnitude diagram. Dynamical models predict the formation of $\mathrm{X}$-shaped bulges as extreme cases of boxy-peanut bulges. However, since $\mathrm{X}$-shaped bulges were known to be present only in external galaxies, models have never been compared to 3D kinematical data for individual stars.

Aims. We study the orbital motion of Galactic bulge stars in the two arms (overdensities) of the $\mathrm{X}$ in the southern hemisphere. The goal is to provide observational constraints to bulge formation models that predict the formation of $\mathrm{X}$-shapes through bar dynamical instabilities.

Methods. Radial velocities have been obtained for a sample of 454 bulge giants, roughly equally distributed between the bright and the faint red clump, in a field at $(l, b)=(0,-6)$. Proper motions were derived for all red clump stars in the same field by combining images from two epochs, which were obtained 11 years apart, with WFI at the $2.2 \mathrm{~m}$ at La Silla. The observed field contains the globular cluster NGC 6558, whose member stars were used to assess the accuracy of the proper motion measurement. At the same time, as a by-product, we provide the first proper motion measurement of NGC 6558. The proper motions for the spectroscopic subsample are analyzed for a subsample of 352 stars, taking into account the radial velocities and metallicities measured from near-infrared calcium triplet lines.

Results. The radial velocity distribution of stars in the bright red clump, which traces the closer overdensity of bulge stars, shows an excess of stars moving towards the Sun. Similarly, an excess of stars receding from the Sun is seen in the far overdensity, which is traced by faint red clump stars. This is explained by the presence of stars on elongated orbits, which are most likely streaming along the arms of the $\mathrm{X}$-shaped bulge. Proper motions for these stars are consistent with qualitative predictions of dynamical models of peanut-shaped bulges. Surprisingly, stars on elongated orbits have preferentially metal-poor (subsolar) metallicities, while the metal rich ones, in both overdensities, are preferentially found in more axisymmetric orbits. The observed proper motion of NGC 6558 has been measured as $\left(\mu_{1} \cos (b), \mu_{\mathrm{b}}\right)=(0.30 \pm 0.14,-0.43 \pm 0.13)$, with a velocity dispersion of $\left(\sigma_{l} \cos (b), \sigma_{b}\right)=(1.8,1.7) \mathrm{mas} / \mathrm{yr}$. This is the first proper motion measurement for this cluster.
\end{abstract}

Key words. Galaxy: bulge - Galaxy: kinematics and dynamics - Galaxy: structure - galaxies: kinematics and dynamics

\footnotetext{
* Based on observations taken with ESO telescopes at the La Silla Paranal Observatory under program IDs 163.O-0741(A), 085.D-0143(A) and 385.B-0735(B) and on observations taken with the Magellan telescope at the Las Campanas Observatory.

$\star \star$ Kinematics for IMACS and FLAMES spectra are only available at the CDS via anonymous ftp to

cdsarc.u-strasbg.fr (130.79.128.5) or via

http://cdsarc.u-strasbg.fr/viz-bin/qcat?J/A+A/555/A91
}

\section{Introduction}

With $\sim 10^{10} M_{\odot}$ in stars (Kent 1992) the Galactic bulge is, after the disk, the second most massive stellar component of the Milky Way. It is the only galactic bulge in which the individual stars can be resolved down to the bottom of the main sequence, allowing the construction of deep color-magnitude diagrams (CMDs), astrometric proper motion (PM) measurements, radial velocities (RVs), and detailed chemical composition studies 
from medium - and high-resolution spectroscopy. Thus, the Milky Way bulge offers a unique opportunity to map the stellar content of a bulge, as summarized by age, metallicity, and kinematical multivariate distributions. All together, this observational evidence should help us to reconstruct the formation history of the bulge, and identify the basic physical processes that have led to its present dynamical structure and stellar content.

At least three distinct processes have been envisaged for the formation of galactic bulges. An early formation by the merging of gas-rich smaller galaxies, which is a process traditionally invoked for the formation of elliptical galaxies (Toomre 1977); a late formation via the secular growth of a bar instability in a pure stellar disk (e.g., Combes \& Sanders 1981; Saha et al. 2010; Shen et al. 2010, and references therein); and, more recently, an early formation via clump instability, migration, and central coalescence in a very gas-rich disk (e.g., Immeli et al. 2004; Förster Schreiber et al. 2006; Carollo et al. 2007; Elmegreen et al. 2008; Bournaud et al. 2009; Genzel et al. 2011). Moreover, secular processes driven by a bar may dynamically change a pre-existing non-rotating bulge into a boxy bulge with high cylindrical rotation (Saha et al. 2012). The predominantly old stellar content of the bulge ( $\gtrsim 10$ Gyr, Ortolani et al. 1995; Zoccali et al. 2003; Clarkson et al. 2011) demands an early formation, hence favoring processes that are supposed to act at an early time. On the other hand, the proven bar shape of the Galactic bulge (Stanek et al. 1994; Dwek et al. 1995; Rattenbury et al. 2007; and references therein) clearly demands that some sort of disk and bar instability had taken place. Thus, it is quite natural to expect that a variety of processes may have contributed to the build up of the bulge.

Although there has been general agreement that the bulge is bar-shaped, some of its structural parameters are still under discussion. Its axial ratios appear to be close to 1:0.35:0.25, but its inclination angle with respect to the line of sight has been reported in the range of $\sim 15$ to 45 degrees, depending on the method used to trace it (e.g., Binney et al. 1997; Dehnen 2000; Bissantz \& Gerhard 2002; Benjamin et al. 2005; Babusiaux \& Gilmore 2005; Rattenbury et al. 2007; Robin et al. 2012; and references therein). Star counts at longitudes $|l|>7$ provided hints for the presence of a second, longer, and thinner bar (e.g., Hammersley et al. 2000; Cabrera-Lavers 2007, 2008; López-Corredoira et al. 2007; Churchwell et al. 2009), which however has been interpreted as more likely being a component of the bar itself (e.g., Martinez-Valpuesta \& Gerhard 2011; Athanassoula 2012).

More recently, McWilliam \& Zoccali (2010) and Nataf et al. (2010) used star counts of red clump (RC) stars from the 2MASS (Skruskie et al. 2006) and OGLE-III (Szymanski et al. 2011) catalogs to show that, along the $l=0$ direction for latitudes exceeding $|b|=5$, the RC splits in two components, indicating the presence of two peaks in stellar density along the line of sight. McWilliam \& Zoccali (2010) interpreted this feature as evidence for the Galactic bar being X-shaped, which was later confirmed by Saito et al. (2011) in a more quantitative analysis of 2MASS RC giants across a larger bulge area. In this context, the main bar is just seen as the inner part of the X-shaped bulge.

$\mathrm{X}$-shaped bulges can be qualitatively produced by some dynamical models as peculiar boxy/peanut (B/P) structures (e.g., Athanassoula 2005; Martinez-Valpuesta et al. 2006; Debattista et al. 2006), and X-shaped isophotes have been observed in a few S0 galaxies. However, such models have been neither compared with observations of velocities for samples of individual stars nor fine-tuned to reproduce a bulge with the characteristics of the Milky Way bulge, at least in part due to lack of observational constraints. We are now beginning to collect kinematical data for a large number of stars in the Galactic bulge, thus setting constraints on the proposed formation mechanisms.

PMs can be obtained for large samples of stars with relatively short exposure times, but a long-time baseline and precise astrometry are required to reach the needed accuracy of a few mas/yr, which corresponds to the bulge PM dispersion. A pioneer study based on photographic plates taken 33 years apart has yielded PMs for 400 stars in Baade's Window (Spaenhauer et al. 1992), later followed by Rich \& Terndrup (1997) in the same field. Zoccali et al. (2001) used the WFPC2 camera on board the Hubble Space Telescope to measure the PM of the bulge globular cluster NGC 6553 as well as the bulge dispersion of PMs in the same field. Feltzing \& Johnson (2002) did likewise for the bulge globular cluster NGC 6528. Vieira et al. (2007) measured PMs in the Plaut Window at $(l, b)=(0,-8)$, whereas a large PM catalog based on OGLE-II data and extending over several fields mostly at $b=-4$, has been obtained by Sumi et al. (2004). This catalog was used by Rattenbury et al. (2007) to compare the PM distribution of RC stars with bulge formation models. Kuijken \& Rich (2002) used the WFPC2 camera on board the Hubble Space Telescope to demonstrate that the bulge CMD can be decontaminated from the foreground disk stars based on the different PM distributions of bulge and disk stars. Following this approach, Clarkson et al. $(2008,2011)$ used two-epoch ACS/HST data to derive a bona fide pure-bulge CMD for a field at $(l, b) \sim(1,-2.51)$, which is clean from disk contamination. They thus demonstrated that the bulge consists of a predominantly old population of $\gtrsim 10 \mathrm{Gyr}$, confirming a similar conclusion by Ortolani et al. (1995) and Zoccali et al. (2003), who used a more crude or a statistical foreground decontamination, respectively.

Important constraints on the bulge formation mechanism come from RV measurements (Rich 1988, 1990; Terndrup et al. 1995; Minniti et al. 1996; Sadler et al. 1996), especially when PMs are available on the same field. In fact, Zhao et al. (1996) combined the PMs by Spaenhauer et al. (1992) with the RVs from most of the above-mentioned samples deriving 3D space velocities for a sample of $62 \mathrm{~K}$ giants, which showed a significant vertex deviation. This result, later confirmed by Soto et al. (2007), indicates a bar-like structure for the Galactic bulge. With a complex, simultaneous analysis of the metallicity distribution function and kinematics of Baade's Window giants, Babusiaux et al. (2010) and Hill et al. (2011) demonstrated that the vertex deviation is mainly produced by the most metal-rich stars, which have disk-like $\alpha$-element abundance (Gonzalez et al. 2011), while the metal-poor, $\alpha$-enhanced stars have a kinematics more typical of a classical spheroid. The BRAVA survey (Howard et al. 2008, 2009; Kunder et al. 2012) obtained RVs for a total of $\sim 10000$ bulge $\mathrm{M}$ giants in several fields spanning a wide range in longitude $(-10<l<+10)$ and latitudes $(b=-4,-6$ and -8$)$, finding evidence for a cylindrical rotation. By comparing those data with their simple $N$-body model, Shen et al. (2010) argued for the Milky Way being a pure disk galaxy, i.e., without the need of a merger-made bulge. It should be emphasized that the vast majority of early-type galaxies (over $~ 86 \%$, cf. Emsellem et al. 2011) are actually fast rotators, with just the most massive elliptical galaxies being predominantly slow rotators. Thus, the cylindrical rotation of the bulge does not necessarily imply a formation mechanism radically different from that of the majority of early-type galaxies.

A different approach of combining metallicity with RV measurements has been followed by Rangwala et al. (2009a,b), who used Fabry-Perot imaging to sample the calcium II triplet (CaT) 
S. Vásquez et al.: 3D kinematics through the X-shaped Milky Way bulge

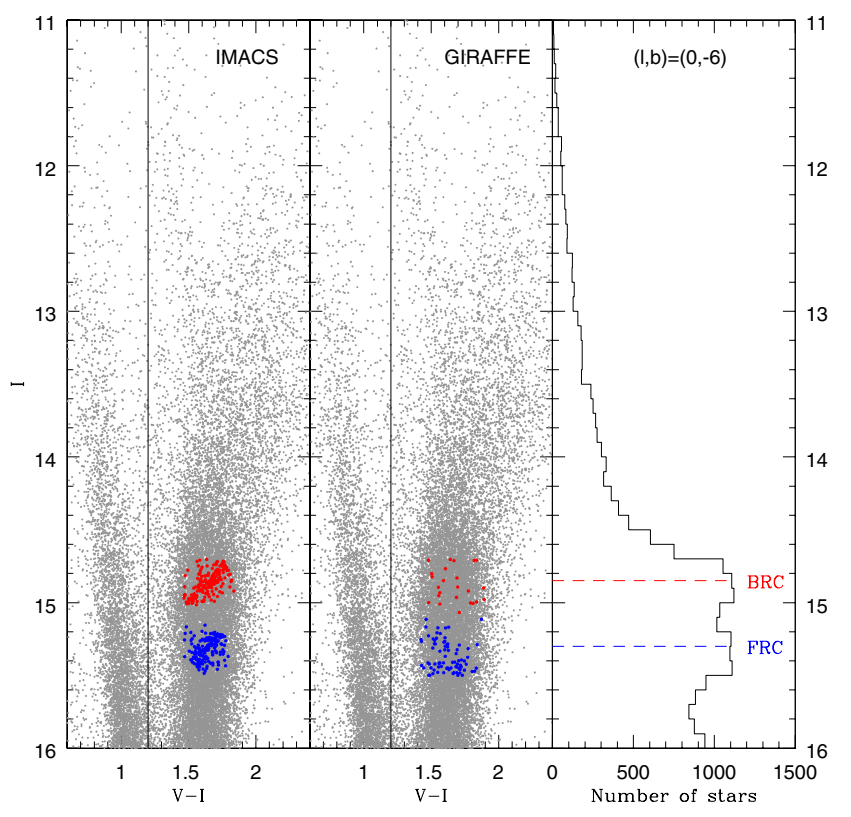

Fig. 1. Left and middle panels: optical CMD of the bulge field at $(l, b)=(0,-6)$ obtained with WFI@2.2 $\mathrm{m}$ at La Silla. The vertical line marks the color cut applied to construct the histogram on the right $(V-I>1.2)$, where the two clumps are clearly visible. The bright red clump (BRC - red points) and faint red clump (FRC - blue points) spectroscopic target selection observed with IMACS on Magellan and with FLAMES-GIRAFFE on VLT are overplotted on the CMD in the left and middle panels, respectively.

lines for $\sim 3400$ stars along the bar $(l=0, \pm 5)$ at $b=-4$. They detected the presence of bar stellar streaming motions along the spanned longitudes. RVs and metallicities separately for bright red clump (BRC) and faint red clump (FRC) stars were first obtained by De Propris et al. (2011) in a bulge field at $(l, b)=(0,-8)$ and more recently by Uttenthaler et al. (2012) in a field at $(l, b)=(0,-10)$ and by Ness et al. (2012) in three fields along the minor axis at $b<-5$.

In the present paper we combine RV and PM measurements to derive the $3 \mathrm{D}$ motion of 454 bulge stars in the BRC and the FRC of a field at $(l, b)=(0,-6)$. Like those in the De Propris et al. study, these stars trace the near and far arms of the X-shaped bulge. The paper is organized as follows. In Sect. 2 we describe the data and reduction methods for our photometric, astrometric, and spectroscopic measures, whereas in Sects. 3 and 4 the resulting RVs and PMs are presented. In Sect. 5 we present the space velocities. The metallicity distributions of the stars in the two RCs are presented in Sect. 6, and conclusions are drawn in Sect. 7.

\section{Observations and data reduction}

\subsection{WFI photometry}

The spectroscopic targets were selected based on the optical $V$, $I$ photometry of a bulge field centered at $(l, b)=(0,-6)$, obtained with the WFI camera at the $2.2 \mathrm{~m}$ telescope at ESO La Silla, on April 15, 1999 as part of the ESO imaging Survey ESO program (EIS, ESO program ID 163.O-0741(A)). The resulting CMD has been already presented in Zoccali et al. (2003) and McWilliam $\&$ Zoccali (2010). Figure 1 shows the bright portion of the $\mathrm{CMD}$, which clearly displays double $\mathrm{RC}$, as illustrated in the right-hand panel. Given the modest dependence of the RC magnitude on stellar population properties (age and metallicity),
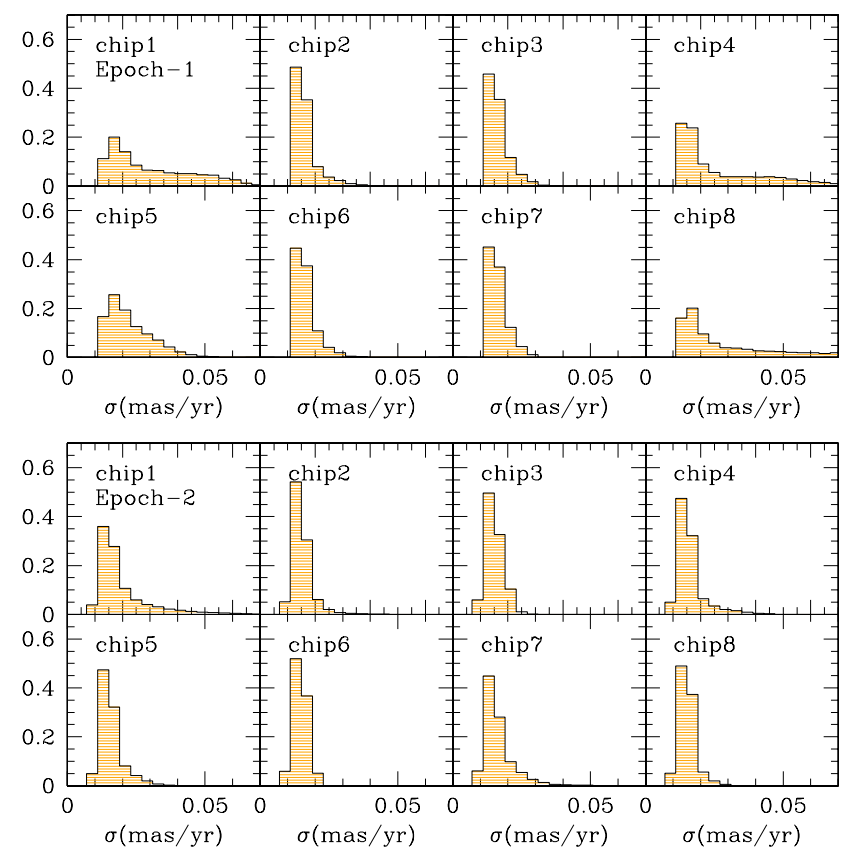

Fig. 2. Dispersion of the position $(X, Y)$ of the stars in every frame, for each epoch, plotted across the WFI detector in units of mas/yr. A higher spread in the dispersion is observed in the edges of the CCD associated with higher order distortion and variable PSF. All of this effects are mostly corrected in the procedure done in the astrometrization (see text for details).

McWilliam \& Zoccali concluded that the two clumps trace two stellar overdensities at different distances along the line of sight and that these overdensities correspond to the near and far arm of the X-shaped bulge. Based on this CMD the red clump targets were selected among the BRC (red dots) and FRC stars (blue dots) for spectroscopic follow-up with Magellan IMACS and VLT FLAMES-GIRAFFE spectrographs.

In order to derive space velocities for our targets, secondepoch images of the same field were obtained on May 6, 2010 with the same instrument and filters as in the first epoch (ESO program ID 085.D-0143(A)). Thus, images were taken through the $V$ - and $I$-band filters, with integration times of $7 \times 50 \mathrm{~s}$ for both. While the seeing of the first epoch was excellent $(0.6-0.7$ arcsec, observed at air mass $=1.06)$, the second epoch had average seeing 1.6 arcsec at air mass $=1.19$. Photometry was carried out with the DAOPHOTII/ALLFRAME packages (Stetson 1988, 1994), on individual chips, while the photometric calibration was derived by comparison of stars with the first epoch.

\subsection{WFI astrometry}

The $X, Y$ coordinates for each star were determined in each individual exposure (hereafter frame) in each epoch. We used seven frames in the first epoch ( $3 \mathrm{~V}$ and $4 \mathrm{I}$, see Zoccali et al. 2003 for details) and 14 frames in the second epoch (7 in $V$ and $I$ ). The set of codes developed by P.B. Stetson (DAOMATCH and DAOMASTER; Stetson, priv. comm.) were used to transform the coordinates of each star in each frame into the system of the reference frame of each epoch. A cubic transformation was allowed among different frames of each epoch in order to properly take into account distortions at the edges of the chips. Different effects contribute to the distortions among different frames and between the two epochs. They include, 
the non-perfect alignment among the chips of the WFI mosaic shown in Fig. 2.7 of the WFI Handbook (version 2.2, March 2013) and the differential atmospheric refraction described in Filippenko (1982). Following Filippenko, we calculated the effect of atmospheric refraction at the central wavelengths of the $V$ and $I$ filters, at the center of the detector, and at one edge (15 arcmin away). The differential shift between the center and the edge varies by 0.002 arcsec between the two filters at the air mass of epoch 1 and by 0.0025 arcsec at the air mass of epoch 2 . This values would not be completely negligible in our case because it corresponds to 0.01 pixels. However, this effect has been removed by the cubic coordinate transformation performed on each chip. An atmospheric effect that cannot be corrected by our procedure is the atmospheric dispersion. Because we are using broadband filters, the refracted light from a star will be dispersed along the parallactic angle, producing a small spectrum instead a point source image on the detector, where maximum light distribution depends on the color of the star. This fact will affect the determination of the spatial centroid, especially for stars with large color differences. To estimate this effect we compute the differential atmospheric refraction as above, for two stars with effective temperatures of $T_{\text {eff }}=4500 \mathrm{~K}$ and $T_{\text {eff }}=6000 \mathrm{~K}$ (which correspond with to a typical RC and MS star). The comparison shows that for both filters the differential shift between the two stars is quite small, reaching $0.001 \mathrm{mas} / \mathrm{yr}$ for the $V$-band and less than $0.0001 \mathrm{mas} / \mathrm{yr}$ for the $I$-band. This scatter on the determination of the coordinates cannot be corrected, but it is so small that it will no affect our analysis.

The final catalog for each epoch was then obtained by averaging the positions of each star in all the frames in which it was detected. Stars in the magnitude range of interest here $(I<16)$ were detected in all the frames of the two epochs, with very few exceptions. An indication of the relative precision of our astrometry is given by the standard deviation, $\sigma$, of the position of a given star in different frames. This is shown in Fig. 2, where histograms of $\sigma=\left(\sigma_{x}^{2}+\sigma_{y}^{2}\right)^{1 / 2}$, in units of mas/yr, are plotted for every WFI chip in both epochs. The dispersion is extremely small for all chips, typically within 0.05 mas/yr, corresponding to 0.002 pixels. We can reach this small dispersion because the photometry has been done with ALLFRAME, a code that "makes simultaneous use of the geometric and photometric information from all the frames of a given field to derive a selfconsistent set of positions and magnitudes..." (Stetson 1994). In other words, the dispersion in Fig. 2 is very small because the individual measurements are not completely independent. Yet it gives an estimate at least of the relative precision in different parts of the mosaic. The figure also shows that the dispersion at the edge of the mosaic (chip \#1, \#4, \#5, and \#8) is higher than in the middle because of the higher order distortion and variable PSF. This effect is larger in the first epoch, most likely because there is a smaller number of frames and the stellar profiles here are slightly undersampled, due to the excellent seeing conditions.

The astrometrization of the final $X, Y$ catalogs was done by means of the IRAF routines ccxymatch, ccmap, and cctran. A seventh-order polynomial transformation was adopted, and the PPMXL catalog (Roeser et al. 2010) was used as a reference. The two astrometrized WFI catalogs were then matched using the topcat catalog-handling package (Taylor 2005), but the residuals showed trends and steps mostly corresponding to the edges of the individual chips. These trends did not disappear by changing the order of the astrometric solution, and they were even larger if the UCAC3 catalog (Zacharias et al. 2010) was used as a reference instead of the PPMXL. A more complex procedure was then adopted to eliminate these residual trends.

First, an astrometric solution (seventh-order polynomial for each chip) was found in order to convert $\left(X_{1}, Y_{1}\right)$ to $\left(\mathrm{RA}_{1}, \mathrm{Dec}_{1}\right)$ and $\left(X_{2}, Y_{2}\right)$ to $\left(\mathrm{RA}_{2}, \mathrm{Dec}_{2}\right)$. This was done using only bulge RGB stars ${ }^{1}$ and the PPMXL catalog as a reference. The two RA, Dec catalogs were then matched to each other. Residual trends were present, as discussed above, but this matched catalog was used only as a first step to combine the chips in a single mosaic and have the star pairs in hand.

A new transformation was then derived between pixel coordinates of star pairs in the two epochs, $\left(X_{1}, Y_{1}\right)$ and $\left(X_{2}, Y_{2}\right)$, and it was applied to the latter to bring them to the pixel coordinate system of the first epoch $\left(X_{2}^{1}, Y_{2}^{1}\right)$. For this transformation, in order to avoid adopting a high-order polynomial that could flare at the edges of the chips, we preferred to divide the field into small boxes $300 \times 300$ pixel wide $(71 \times 71$ arcsec $)$ and impose a first-order transformation (a plane) in each subfield. Finally, both $\left(X_{1}, Y_{1}\right)$ and $\left(X_{2}^{1}, Y_{2}^{1}\right)$ were transformed to (RA, Dec) using the same astrometric solution found in the first step, for epoch 1 only. This last step ensured that any spurious trend in the astrometric solution was applied to the pixel coordinates of both epochs, which was effective in completely removing the trends in the residual differences between the coordinates of the two epochs, thus allowing us to minimize the PM error. The final (RA, Dec) of both epochs were transformed into galactic coordinates $(l, b)$ by means of the topcat package.

\subsection{IMACS spectra}

Spectra for 177 BRC stars and 175 FRC stars were observed with the multislit mode of the IMACS spectrograph at the Las Campanas Observatory on July 10, 2010. A 1200 line/mm grating with a blaze angle of 26.7 degrees was used. This setup produces spectra centered at $\sim 8500 \AA$ (the precise value depending on the position of the star in the field of view) with a resolution of $R \sim 5000$. The spectra have $S / N \sim 40$ in the CaT region, yielding velocities accurate to a few $\mathrm{km} \mathrm{s}^{-1}$.

The spectra were reduced using the COSMOS pipeline, provided by The Carnegie Observatories. This pipeline processes the multislit spectra from IMACS and applies bias and flat field corrections, wavelength calibration, and sky subtraction to each $2 \mathrm{~d}$ spectrum. The final extraction to $1 \mathrm{~d}$ spectra and velocity measurement was done with IRAF apall and fxcor tasks. As a test for the wavelength calibration made for COSMOS, the sky lines in each spectra were cross-correlated with a sky line template from UVES (Hanuschik 2003). The residual shifts found $\left(\sim|10| \mathrm{km} \mathrm{s}^{-1}\right)$ were applied to individual spectra in order to set them at the geocentric rest frame.

The RVs of our sample were measured by cross-correlation against a synthetic spectral template for a typical RGB star with $T_{\text {eff }}=4750 \mathrm{~K}$ and $[\mathrm{Fe} / \mathrm{H}]=-1.3$ covering the region from $8350 \AA$ until $8950 \AA$, where the CaT lines are located. Due the IMACS CCD mosaic configuration, with eight chips in a square array separated by gaps, in a few spectra one of the three lines fell in the gap. In such cases the measurement was made using only the available section in the spectra and the respective range in the template. The typical error obtained from the cross-correlation was $\sim 2.0 \mathrm{~km} \mathrm{~s}^{-1}$ without significant outliers

1 Only stars with $V-I>1.4$ and $V<18.5$ were used, and the disk $\mathrm{RC}$ sequence sticking out from the bulge RGB at $V-I=1.6, V=15$ upward and to the blue was also excluded. 
$\left(\sigma \sim 0.8 \mathrm{~km} \mathrm{~s}^{-1}\right)$. Finally, heliocentric corrections were calculated with the IRAF task rvcorrect and applied to all RVs.

\subsection{FLAMES-GIRAFFE spectra}

The RC stars in the same field at $(l, b)=(0,-6)$ were also observed with the multifiber FLAMES-GIRAFFE spectrograph at the VLT in Medusa mode within the ESO program ID 385.B0735(B) in service mode. Spectra were taken with the LR08 setup, centered at $8817 \AA$ and yielding a spectral resolution of $R=6500$ and average $S / N \sim 50$. These observations were not especially fine tuned to discriminate between the two RCs, and therefore the target stars were not evenly distributed between the two RCs. Of 130 Medusa fibers 24 were allocated to the BRC and 78 to the FRC stars. The middle panel in Fig. 1 shows the FLAMES-GIRAFFE target selection in the CMD.

The GIRAFFE spectra were extracted and wavelength calibrated with the instrument pipeline available from ESO, and RVs were measured via cross-correlation with IRAF fxcor task, using the same template set as for IMACS spectra. As expected by the difference in resolution from the usage of fiber vs. slit spectrograph, the typical error for GIRAFFE RV measurements was smaller than for IMACS observations ( $V_{\text {Helio }}$ error $\sim 1 \mathrm{~km} \mathrm{~s}^{-1}$ with dispersion $\sim 0.3 \mathrm{~km} \mathrm{~s}^{-1}$ ).

There are four stars in common between IMACS and GIRAFFE observed data set. These stars have a mean difference on measured RV of $\Delta V_{\text {Helio }} \sim 4 \pm 2 \mathrm{~km} \mathrm{~s}^{-1}$ and were used to measure the systematic difference in $[\mathrm{Fe} / \mathrm{H}]$ for both samples (Sect. 6).

\section{Radial velocities for bulge red clump stars}

Figure 3 (top) shows the cumulative heliocentric RV distribution for the RC stars obtained in this work, compared with the sample of K giants stars analyzed in Zoccali et al. (2008; see also Babusiaux et al. 2010) and with the sample of $M$ giants observed within the BRAVA survey (Kunder et al. 2012). The three samples were obtained in the same field at $(l, b)=(0,-6)$, and the number of stars in each sample is indicated on the labels. According to a Kolmogorov-Smirnov test, RC stars and M giants have the same RV distribution, with a confidence of $71 \%$. On the other hand, $\mathrm{K}$ giants, selected in a box located at $\sim 0.75 \mathrm{mag}$ above the RC, have an RV distribution more peaked towards $V_{\text {Helio }}=0 \mathrm{~km} \mathrm{~s}^{-1}$, which is different from that of RC stars with a confidence of $89 \%$. This difference is also clear if we measure the RV dispersion of the two samples: $\sigma_{\mathrm{RC}}=97 \pm 4 \mathrm{~km} \mathrm{~s}^{-1}$ versus $\sigma_{\mathrm{K}}=83 \pm 4 \mathrm{~km} \mathrm{~s}^{-1}$. The RV dispersion of $\mathrm{M}$ giants from the BRAVA sample is $\sigma_{\mathrm{M}}=101 \pm 5 \mathrm{~km} \mathrm{~s}^{-1}$, within $1 \sigma$ of that of RC stars.

One possible explanation for the difference between the $\mathrm{RV}$ distribution of $\mathrm{RC}$ and $\mathrm{K}$ giants in this field is a different amount of contamination by foreground disk stars, which are expected to have an RV distribution peaked at $V_{\text {Helio }}=0 \mathrm{~km} \mathrm{~s}^{-1}$. Indeed, at different distances and reddenings along the line of sight, the disk RC is clearly visible in the CMD of Fig. 1 as a diagonal sequence parallel to the disk MS, but shifted $\sim 1$ mag to the red. This sequence intersects the bulge RGB precisely between $I=14$ and $I=14.5$, which are the magnitude limits of the K giants target box (cf., Fig. 1 in Zoccali et al. 2008). In contrast, $\mathrm{M}$ giants in the BRAVA sample were selected so as to avoid the disk RC sequence, while bulge RC stars largely outnumber the disk foreground stars in the same CMD box because they clump at that magnitude.

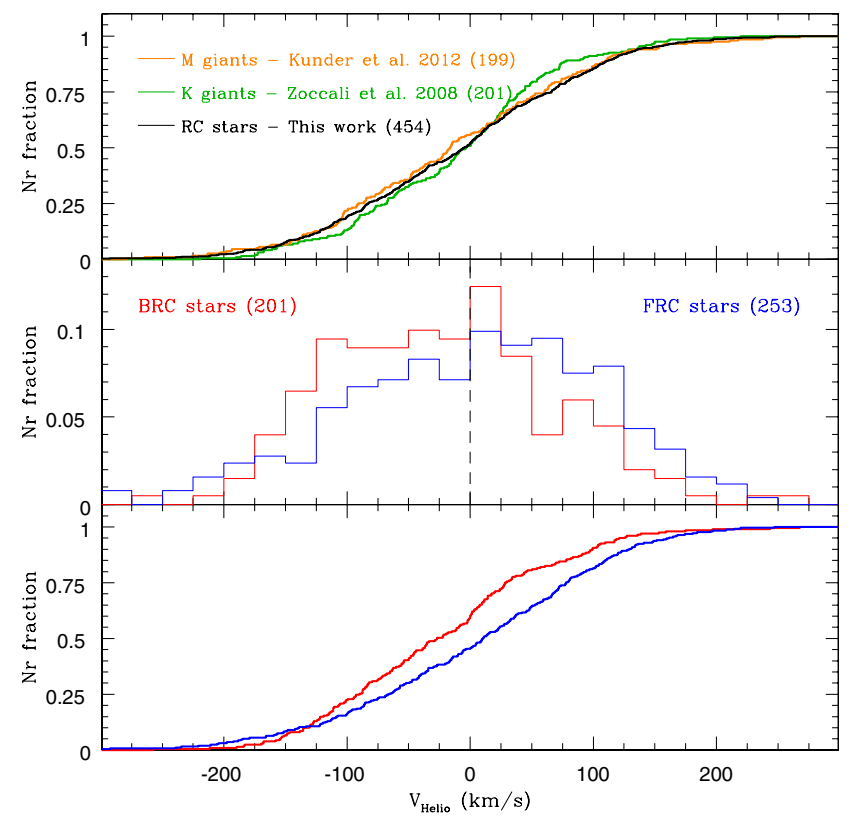

Fig. 3. Upper panel: cumulative distributions of the heliocentric radial velocities for bulge stars in the RC (black; this work), K giants (green; Zoccali et al. 2008) and M giants (orange; Kunder et al. 2012). All the stars are located in the same field at galactic coordinates $(0,-6)$. A Kolmogorov-Smirnov test shows that RC stars and $\mathrm{M}$ giants have radial velocity distributions that are similar, with a confidence of $71 \%$. In contrast, $\mathrm{K}$ giants have a radial velocity distribution that is more peaked at $V_{\text {Helio }}=0 \mathrm{~km} \mathrm{~s}^{-1}$ with $89 \%$ confidence of being different from RC stars. See text for discussion. Middle and lower panels: histograms and cumulative distributions for heliocentric radial velocities of bulge stars in the BRC and FRC, respectively. The two distributions differ from each other, with a confidence of $99.95 \%$, according to the Kolmogorov-Smirnov test.

The middle and lower panels of Fig. 3 show, respectively, the differential and cumulative heliocentric RV distribution of stars in the BRC and in the FRC. Both the IMACS and GIRAFFE target stars are included in the histograms. The KolmogorovSmirnov test yields a confidence of $99.95 \%$ that the two distributions are different. In fact, it is visually evident that there is an excess of stars with $V_{\text {Helio }} \sim-80 \mathrm{~km} \mathrm{~s}^{-1}$ in the BRC sample, while a similar excess is visible at $V_{\text {Helio }} \sim+80 \mathrm{~km} \mathrm{~s}^{-1}$ in the FRC sample. A dashed line at $V_{\text {Helio }}=0 \mathrm{~km} \mathrm{~s}^{-1}$ has been drawn in these panels to visually emphasize the asymmetry of the two distributions.

Before trying to understand the observed asymmetry in the RV distribution of the two clumps, it is worth considering two sources of contamination. First of all, we know that foreground disk RC at distances $d>5 \mathrm{kpc}$ from the Sun would fall into the CMD selection boxes. These stars should have an $\mathrm{RV}$ close to zero, and a narrower velocity dispersion, especially those closer to the Sun, contaminating the BRC. According to the Besançon model of the Galaxy (Robin et al. 2003), the contamination from disk foreground stars should be small for both RCs (3\% and $8 \%$ by thin and thick disks, respectively). The density of both disks in the inner few kpcs of the Galaxy, however, has never been constrained observationally, so the model predictions are extremely uncertain on this point. Recently, the Besançon model was updated (Robin et al. 2012), including two populations in the inner bulge. Despite the differences with the old model (both for the bulge component and the adjustments to the disks, most notably the thick disk), the figures for the contaminations are similarly low: $3 \%$ and $7 \%$ contamination 
from the thin and thick disk, respectively, for the $\mathrm{BRC}$, while a contamination of $4 \%$ and $7 \%$ were estimated for the FRC. In order to estimate the contamination fractions quoted above we split (arbitrarily) the single RC predicted into two components, each one containing half of the bulge RC stars, and counted the model disk stars in two boxes with the magnitude and color limits of our spectroscopic targets. Overall, we can expect that the stars at RV close to zero might suffer from some degree of disk contamination, which is hard to quantify here.

A second source of contamination comes from the bulge RGB stars in the two overdensities along the line of sight. This is because the upper RGB stars of the far arm (that corresponds to the FRC) overlap the BRC, whereas the lower RGB stars of the nearby arm contaminate the FRC selection box. Furthermore, other bulge stars not associated with the two arms (overdensities) might also exist along the line of sight.

All together, these contaminations and cross-contaminations act in such a way that each selection box on the CMD will not include purely stars at the distances of the overdensities identified by the two RCs, with the effect of smoothing the features of the two RV distributions seen in Fig. 3 and making them more similar to each other than they actually are.

An estimate of the fraction of RGB stars included in each of the two RC boxes has been calculated by generating a synthetic CMD from the BASTi library of stellar models, as well as assuming an age of $10 \mathrm{Gyr}$ and solar metallicity. Then the crosscontamination was evaluated considering the $0.5 \mathrm{mag}$ difference in distance modulus $(\sim 2 \mathrm{kpc})$ between the two overdensities and counting the fraction of RGB and RC stars that fall in each selection box. After rescaling these two fractions by taking the cone effect into account, we finally estimated a cross-contamination fraction of $\sim 18 \%$ in both RCs.

It is important to note that the above-mentioned effects, namely, foreground and cross-contamination, are certainly present, even if their impact is hard to precisely quantify here. This consideration supports the assumption that we make in the following analysis, according to which the near arm is dominated by stars with mostly negative RVs, roughly centered at $V_{\text {Helio }}=-80 \mathrm{~km} \mathrm{~s}^{-1}$, whereas the far arm is dominated by stars with mostly positive RVs, roughly centered at $V_{\text {Helio }}=$ $+80 \mathrm{~km} \mathrm{~s}^{-1}$.

\section{Proper motions}

In order to assess the precision of the PMs derived as described in Sect. 2.2 we took advantage of the presence in our $(l, b)=$ $(0,-6)$ field of the globular cluster NGC 6558. To this end, we select the stars within a radius of 2 arcmin from the cluster center (half-light radius $\sim 2.15$ arcmin, taken from Harris 1996). Due to the lower metallicity of NGC $6558([\mathrm{Fe} / \mathrm{H}]=-0.97 \pm 0.15)$ with respect to the bulge, it is possible to select a clean sample of cluster stars from the observed CMD selecting stars on the upper RGB $(I<16.5)$ that are bluer than the bulge RGB. Cluster stars are expected to have an internal velocity dispersion of $\sim 10 \mathrm{~km} \mathrm{~s}^{-1}$ (Pryor \& Meylan 1993), which, at the distance of NGC 6558 corresponds to a PM dispersion of $0.3 \mathrm{mas} / \mathrm{yr}$, too small to be measured here. Therefore, we attribute the observed dispersion of cluster stars completely to observational errors. After a 3-sigma clipping, cluster stars show an observed mean PM of $\left(\mu_{1} \cos (b), \mu_{b}\right)=(0.30 \pm 0.14,-0.43 \pm 0.13)$, with a dispersion of $\left(\sigma_{l} \cos (b), \sigma_{b}\right)=(1.8,1.7) \mathrm{mas} / \mathrm{yr}$. The latter is, therefore, the observational error we will use to deconvolve the observed PM dispersion of bulge stars. We note that this is an upper limit to our real error because cluster stars are more crowded
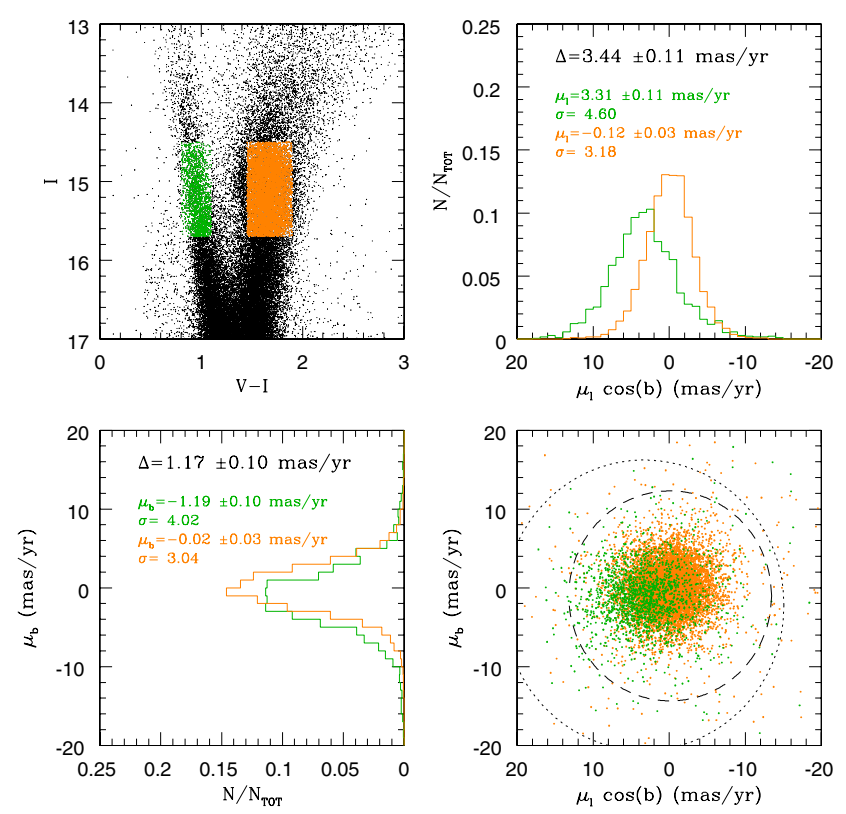

Fig. 4. PMs measured at $(l, b)=(0,-6)$ field. RGB bulge stars and disk dwarfs are shown with orange and green colors respectively. Dotted and dashed circles in $\mu_{l} \cos (b)$ vs. $\mu_{b}$ plot show the $3 \sigma$ data selection used for disk and bulge stars, respectively. The PM distribution derived from this selection shows a systematic difference between disk and bulge population, in agreement with Clarkson et al. (2008).

than bulge stars and because NGC6558 happens to fall at the edge of chip \#6.

Another test to assess the accuracy of our catalog is to separate a pure disk population, selected on the blue main sequence in the CMD (as shown in Fig. 4) and the bulge population selected along the RGB near the RC. The PM diagram for these two samples shows that there is a difference between the mean PM of the disk and bulge populations. After applying a 3-sigma clipping to both distributions, the disk-bulge offset was found to be $\left(\Delta \mu_{1} \cos (b), \Delta \mu_{b}\right)=(3.44 \pm 0.11,1.17 \pm$ $0.10) \mathrm{mas} / \mathrm{yr}$, which compares favorably to $(3.21 \pm 0.15,0.81 \pm$ 0.13 ) found by Clarkson et al. (2008) in a field at $(l, b)=$ $(1.25,-2.65)$. Additionally, if we deconvolve the bulge PM dispersion from the observational error as estimated above, we obtain $\left(\sigma_{l} \cos (b), \sigma_{b}\right)=(2.62,2.52) \mathrm{mas} / \mathrm{yr}$, in excellent agreement with the values of $\left(\sigma_{l} \cos (b), \sigma_{b}\right)=(2.64,2.40) \mathrm{mas} / \mathrm{yr}$ measured by Rattenbury et al. (2007) in two fields at $(l, b)=$ $(-0.25,-5.70)$ and $(l, b)=(-0.14,-5.91)$.

The two epoch images analyzed here had not been obtained with the purpose of deriving PMs. Therefore the observational strategy (dithering, etc.) was not optimized for this kind of study. Nevertheless, the measurements reported above confirmed that the derived PMs, although not very precise, should be good enough to allow us to identify differences in the mean PM of the two bulge overdensities traced by the BRC and FRC.

The PM of stars in the two RCs is shown in Fig. 5. The BRC contains stars with $14.5<I<15.0$ and $1.35<V-I<2$, while the FRC stars have $15.2<I<15.7$ and $1.35<V-$ $I<2$, as illustrated in the upper left panel of Fig. 5, where the two groups have approximately the same number of stars. There is a small but statistically significant shift $\Delta \mu_{1} \cos (b)=0.79 \pm$ $0.08 \mathrm{mas} / \mathrm{yr}$ in the longitude PMs between the two distributions ( $\sim 100 \%$ confidence from a Kolmogorov-Smirnov test), whereas the two latitude PM distributions are barely distinguishable. 
S. Vásquez et al.: 3D kinematics through the X-shaped Milky Way bulge
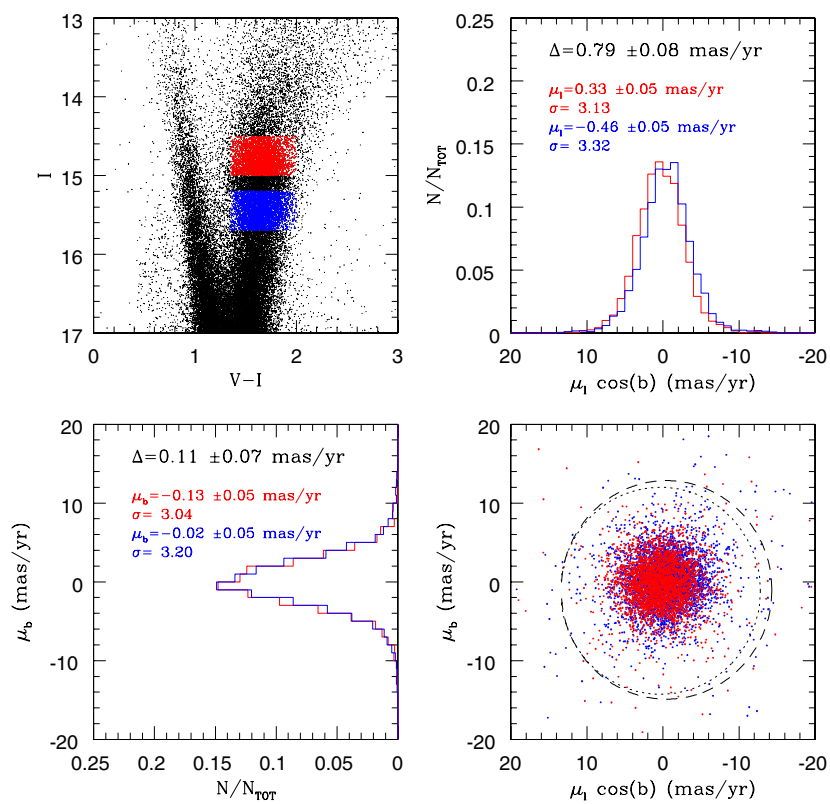

Fig. 5. PM measured at $(l, b)=(0,-6)$ field for bulge RC stars. The blue and red dots identify the selected BRC and FRC stars on the CMD, respectively. A $3 \sigma$ selection (lower right panel) in the $\mu_{l} \cos (b)$ and $\mu_{b}$ plane is used to define the sample further analyzed with histograms. The lower left panel shows $\mu_{b}$ distribution, while the upper right panel compares $\mu_{l} \cos (b)$ distributions for the BRC and FRC stars. The mean values of $\mu_{l} \cos (b)$ and $\mu_{b}$ for the BRC and FRC samples are given in the respective panels showing the distributions, and we also provide the mean differences in proper motion for the two RC populations.

For the spectroscopic targets it is possible to combine the information from the PMs with that from RVs, thus deriving 3D space velocity for individual stars. The upper panels of Fig. 6 show that both BRC and FRC stars share a common trend in $\mu_{1} \cos (b)$ vs. $V_{\text {Helio, }}$, which is not observed in $\mu_{b}$ (Pearson correlation coefficient of $\sim-0.2$ and $\sim 0.05$, respectively). To emphasize this behavior a linear regression between PMs and RV was calculated for each sample and overplotted on the data points.

Following the arguments given in Sect. 3, the cleanest sample of BRC stars is the one with negative RVs, while the cleanest sample of FRC stars has positive RVs. If these arguments are correct, then the stars we want to probe should be at the extreme of the two RV distributions. Arbitrary selections of stars with $V_{\text {Helio }}<-50 \mathrm{~km} \mathrm{~s}^{-1}$ and $V_{\text {Helio }}>+50 \mathrm{~km} \mathrm{~s}^{-1}$ were made to the BRC and FRC, respectively, and they are shown at the bottom of each scatter plot, where small versions of the RV distributions are also shown. The PMs of these stars are compared in the two lower panels in the same figure, showing a significant offset in $\mu_{1} \cos (b)$, while the mean $\mu_{b}$ values are virtually identical.

These results can be interpreted as follows. In the near and far overdensities, we observe stars on radial orbits ( $V_{\text {Helio }}$ nonzero). An excess of stars approaching the Sun is present in the near overdensity, while an excess of stars receding from the Sun is present in the far overdensity. By itself, this is an indication of stars in streaming motions along the bar. In our bulge the bar has been proved to be X-shaped. Structures of this kind have been proposed to form by buckling and bending of the stellar distribution where stars are rearranged on banana orbits, i.e., the family of $x 1 v 1$ orbits according to Patsis et al. (2002) that look either like a "frown" $(\frown)$ or a "smile" $(\smile)$ when viewed edge on. This is the main family of stable, periodic orbits forming a peanut-shaped or X-shaped bulge.
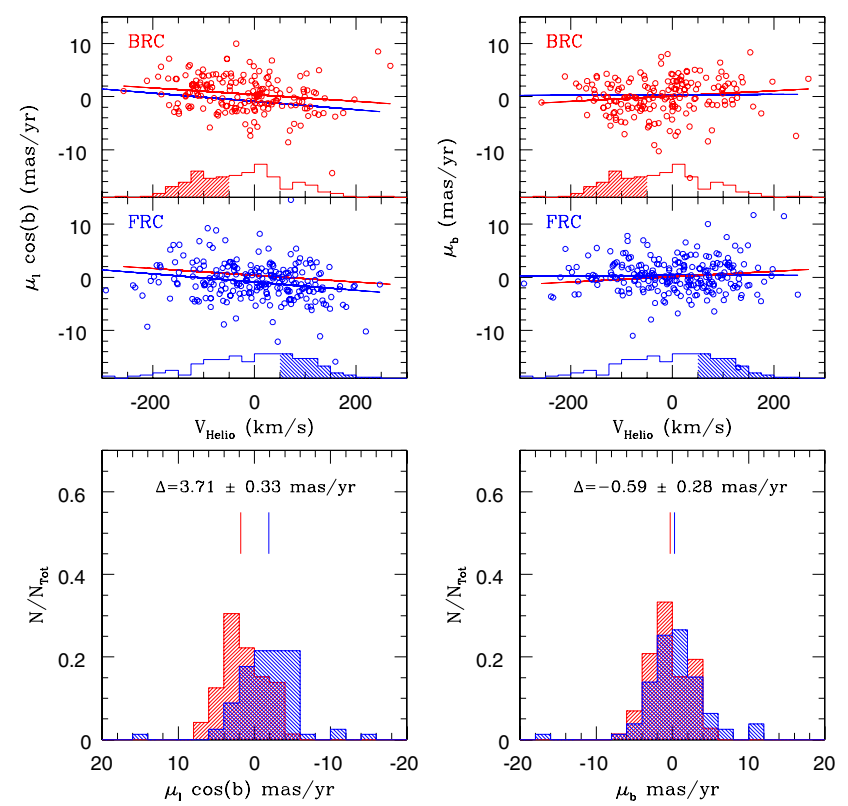

Fig. 6. Upper panels: PMs versus RVs for BRC and FRC sample. Color lines (red for BRC and blue for FRC) shows the linear regression for our data in the respective sample. As is shown in the right upper panel, no significant difference in $\mu_{b}$ versus RV is observed, while for $\mu_{l} \cos (b)$ a common trend with RV is found (left upper panel). Under each scatter plot is also shown the scaled RV distribution for each sample to define a pure BRC and FRC sample (red and blue dashed regions, respectively). Lower panels: histograms for observed proper motions and the difference in the median for selected stars in each pure sample defined above.

Stars near the velocity inversion points of such orbits, which are the ones that we are probing at $b=-6$, are expected to go in opposite radial, and opposite longitude directions if, as in our case, the line of sight crosses two opposite sides of the orbit $^{2}$. However, since both overdensities at this latitude lie below the plane, they are expected to share a common mean $\mu_{b}$, as observed.

Our result differs from that of De Propris et al. (2011), who found no differences between the RV distribution of BRC and FRC stars in a fields at $b=-8$. Although we do not have a conclusive explanation for this discrepancy, a few factors can account for at least part of it. De Propris et al. (2011) selected their targets from the 2MASS CMD. It is clear from their Fig. 1 that they have larger contamination from disk main-sequence stars in the FRC and possibly also from the BRC (due to the larger error on individual magnitudes of 2MASS versus WFI photometry). A slightly larger error on the magnitudes of individual stars in 2MASS versus WFI photometry and a slightly larger error on the RVs (from their lower $\mathrm{S} / \mathrm{N}$ and lower resolution spectra) might also contribute to mask out the features we found in the FRC distribution.

RVs for stars in the two RCs visible on the bulge minor axis were recently also derived by Uttenthaler et al. (2012) and Ness et al. (2012). The latter compares the measured distributions with the prediction of a bulge model by Athanassoula (2003). The model was not optimized to reproduce the Galactic bulge and hence cannot be taken quantitatively, but it is a model of a peanut-shaped bulge that, at least qualitatively, reproduces the two observed overdensities along the lines of sights on the minor axis. The model predicts the presence of a clear asymmetry in the

2 The line of sight at $(l, b)=(0,-6)$ intercepts the two overdensities formed by the family of frown-like orbits. In particular, it touches the west side of the near part of the frown and the east side of the far one. 


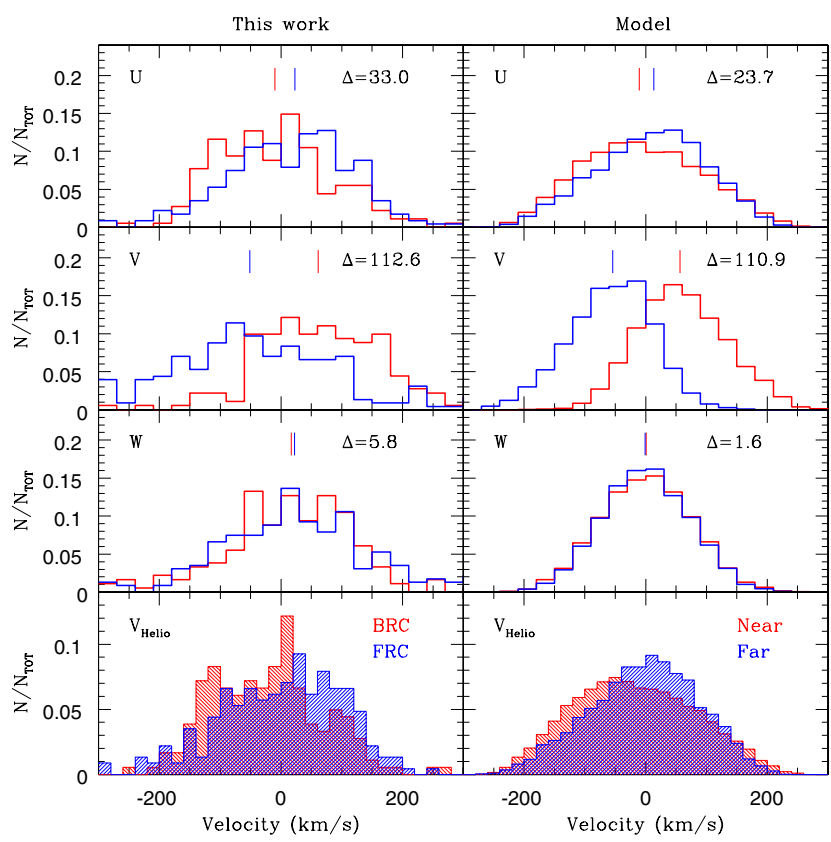

Fig. 7. BRC and FRC are compared with respect to the kinematical model for a strong boxy-peanut bulge (Debattista et al. 2005). From the model, two samples were selected from the two overdensities formed by the near (red) and far (blue) arms of the boxy-peanut stellar distribution, in the line of sight for $(l, b)=(0,-6)$. Color lines over $U, V$, and $W$ histograms correspond to the median value for each distribution.

RV distribution at $b=-5$, similar to the one observed by Ness et al. (2012) at this latitude and to the one presented here. At larger latitudes, however, the asymmetry becomes much weaker in the model and in the observations by Ness et al. (2012) at $b=-7.5$ and $b=-10$. The fact that the asymmetry is predicted to weaken at high latitudes might explain the similarity between the RV distributions of BRC and FRC observed by De Propris et al. (2010) and Uttenthaler et al. (2012).

We can conclude that we have identified stars in the near and far overdensities of the lower part of the X-shaped bulge, whose $3 \mathrm{D}$ kinematics are qualitatively consistent with the prediction of dynamical models producing a peanut-shaped or an X-shaped bulge from the buckling instability of a bar. These are not all the stars we see in the near and far overdensities traced by the near and far RCs. In each of the two RCs, there are indeed also stars sharing the same 3D velocities, as well as a small number of stars with RVs opposite to the main stream. These can be qualitatively interpreted as stars in more stochastic (spheroid-like) orbits, plus some degree of cross-contamination (cf. Sect. 3).

\section{Space velocities}

Space velocities were obtained for all the spectroscopic targets in the $U, V, W$ Galactic Cartesian system following Johnson \& Soderblom (1987), assuming that stars in the BRC and FRC are on average at a distance of 6.5 and $8.5 \mathrm{kpc}$ from the Sun, respectively. Because our PMs are relative to the mean position of the bulge stars instead of quasars or distant galaxies, it is necessary to correct them by the relative motion of the galactic center with respect to an inertial frame before computing $U, V$, and $W$ velocities. Sumi et al. (2004) determine this correction as ${ }^{3}$

$$
\begin{aligned}
\mu_{\alpha * \mathrm{INERT}} & =\mu_{\alpha * \mathrm{OBS}}-\mu_{\alpha * \mathrm{GC}}+\mu_{\alpha * \mathrm{GC}, \mathrm{INERT}} \\
\mu_{\delta_{\mathrm{INERT}}} & =\mu_{\delta \mathrm{OBS}}-\mu_{\delta_{\mathrm{GC}}}+\mu_{\delta \mathrm{GC}, \mathrm{INERT}},
\end{aligned}
$$

\footnotetext{
${ }^{3} \mu_{\alpha *}=\mu_{\alpha} \cos \delta$.
}

where the subscript OBS and GC refer to observed PM of individual stars and the mean PM of the Galactic bulge respectively. The quantities $\left(\mu_{\alpha * \mathrm{GC}, \mathrm{INERT}}, \mu_{\delta_{\mathrm{GC}, \mathrm{INERT}}}\right)=(-2.93,-5.17)$ mas/yr correspond to the expected motion of the galactic center with respect to the inertial frame. The spatial velocities were corrected from the peculiar solar motion $(u, v, w)_{\odot}=$ (11.112.24, 7.25) $\mathrm{km} \mathrm{s}^{-1}$ (Schönrich et al. 2010), considering the solar circle radius and local standard of rest as $R_{\mathrm{GC}}=8.0 \mathrm{kpc}$ and $\mathrm{LSR}_{\odot}=220 \mathrm{~km} \mathrm{~s}^{-1}$, respectively. We adopted the usual notation where $U$ is the radial component along the star-Galactic center direction, positive towards the Galactic center; $V$ is the peculiar rotational velocity of each star with respect to its local standard of rest, positive in the sense of the Galactic rotation; $W$ is the vertical velocity component, positive towards the north Galactic pole.

\subsection{Model comparison}

We compare our observations to the high-resolution model R1 of Debattista et al. (2005). Here we briefly describe this model and the method we used for comparing with the observational data. This model starts out with an exponential disk with a ratio of scale height to scale length, $z_{\mathrm{d}} / R_{\mathrm{d}}=0.05$. The disk has a Toomre- $Q=1.2$ and is immersed in an isothermal halo with scale-length $r_{\mathrm{h}}=3.3 R_{\mathrm{d}}$. The model has $7.5 \times 10^{6}$ particles; it was evolved on a cylindrical grid code described in Sellwood \& Valluri (1997). The model forms a bar and experiences a violent buckling instability leading to the formation of a B/P-shape. The formation of the $\mathrm{B} / \mathrm{P}$-shape is presented as an animation in Debattista et al. (2006), where it is referred to as model L2.

In order to compare the model with the Milky Way, we need to rescale from natural units in which $R_{\mathrm{d}}=M_{\mathrm{d}}=G=1$ (where $M_{\mathrm{d}}$ is the disk mass) to physical units and rotate the model to reproduce the observed inclination bar in the Galaxy. To scale sizes, we use units of the bar radius, placing the observer at two bar radii from the center of the galaxy, with the bar extending to $\sim 1.45 R_{\mathrm{d}}$ of the initial disk. We scale velocities by multiplying the unit velocity by $250 \mathrm{~km} \mathrm{~s}^{-1}$. The bar in the model was rotated to an angle of 20 degrees relative to the Sun-Galactic center direction. We selected particles from both sides of the center of the model, at an observing window of $(l, b)=(0,-6)$. The selection of particles was done based on their spatial distribution along the $x$ coordinate. Two overdensities were observed, corresponding with the two arms of the " $\mathrm{X}$ " (B/P-shape). Around each peak, near and far samples were defined in order to better represent the position of the clumps, avoiding background and foreground contamination. Figure 7 shows a qualitative comparison between the observations and the model, in which the median values for the BRC/near and FRC/far samples and in particular their differences are consistent. Table 1 shows the mean, dispersion, and median for each velocity component present in Fig. 7. As can be observed, most of the velocities predicted by the model are consistent with the observed ones (within the errors) except for $\mathrm{W}$, which is hotter than the model in $\sim 20 \mathrm{~km} \mathrm{~s}^{-1}$. A mismatch in the specific value of the mean and dispersion for the velocity distributions (instead the difference) is not unexpected because the model was not been built specifically for the Milky Way. Additional analysis will be presented in Gardner et al. (in prep.).

It is worth emphasizing that this consistency of our data with the model does not necessarily prove that the model fully represents the formation history of the Milky Way bulge. Indeed, in this class of purely $N$-body models (e.g., Debattista et al. 2006; Shen et al. 2010), the gas contribution of the overall event of 
Table 1. Statistics for velocities presented in Fig. 7 in $\mathrm{km} \mathrm{s}^{-1}$.

\begin{tabular}{|c|c|c|c|c|c|c|c|c|}
\hline & $V_{\text {Helio }}$ & $U$ & V & $W$ & $V_{\text {Helio }}$ & $U$ & $V$ & $W$ \\
\hline & $\operatorname{BRC}(N=181)$ & & & & $\operatorname{FRC}(N=227)$ & & & \\
\hline $\begin{array}{l}\text { Mean } \\
\sigma \\
\text { Median } \\
\end{array}$ & $\begin{array}{c}-22.3 \pm 6.9 \\
62.1 \pm 4.9 \\
-21.4 \\
\end{array}$ & $\begin{array}{c}-10.0 \pm 7.0 \\
64.5 \pm 4.9 \\
-9.9 \\
\end{array}$ & $\begin{array}{c}65.9 \pm 8.2 \\
68.5 \pm 5.8 \\
61.4 \\
\end{array}$ & $\begin{array}{c}13.8 \pm 8.4 \\
66.4 \pm 5.9 \\
16.8 \\
\end{array}$ & $\begin{array}{c}1.0 \pm 6.7 \\
72.3 \pm 4.8 \\
9.5 \\
\end{array}$ & $\begin{array}{c}15.0 \pm 6.8 \\
70.4 \pm 4.8 \\
23.1 \\
\end{array}$ & $\begin{array}{c}-42.6 \pm 10.4 \\
86.3 \pm 7.3 \\
-51.2 \\
\end{array}$ & $\begin{array}{c}26.6 \pm 8.9 \\
77.1 \pm 6.3 \\
22.6 \\
\end{array}$ \\
\hline $\begin{array}{l}\text { Mean } \\
\sigma \\
\text { Median }\end{array}$ & $\begin{array}{c}\text { Near }(N=9590) \text { : } \\
-17.0 \pm 1.0 \\
74.0 \pm 0.7 \\
-21.7\end{array}$ & $\begin{array}{c}-5.9 \pm 1.0 \\
72.0 \pm 0.7 \\
-10.3\end{array}$ & $\begin{array}{c}60.4 \pm 0.7 \\
49.4 \pm 0.5 \\
56.4\end{array}$ & $\begin{array}{c}0.6 \pm 0.8 \\
51.9 \pm 0.5 \\
0.9\end{array}$ & $\begin{array}{c}\text { Far }(N=7349): \\
-2.7 \pm 1.0 \\
61.3 \pm 0.7 \\
1.5\end{array}$ & $\begin{array}{c}8.1 \pm 1.1 \\
63.8 \pm 0.7 \\
13.4\end{array}$ & $\begin{array}{c}-57.7 \pm 0.8 \\
46.8 \pm 0.6 \\
-54.5\end{array}$ & $\begin{array}{c}-0.2 \pm 0.8 \\
49.2 \pm 0.6 \\
-0.7\end{array}$ \\
\hline
\end{tabular}

bulge formation is not taken into account. Instead, when the bulk of the bulge stars formed some $\sim 10$ Gyr ago, the Galaxy was likely to be very gas rich, with a gas fraction of $\sim 50 \%$, as indicated by direct observations of star-forming galaxies at the corresponding lookback time (i.e., at $z \simeq 2$, see Tacconi et al. 2010; Daddi, et al. 2010).

\section{Metallicity distribution}

The CaT metallicities for both the IMACS and the GIRAFFE spectra were derived using the calibration by Vásquez et al. (in prep.). The latter was constructed by observing a sample of RC and RGB stars in Baade's Window, with the GIRAFFE spectrograph in the LR08 setup centered at $8817 \AA$. GIRAFFE highresolution spectra had been previously obtained for the same stars by our group, and $[\mathrm{Fe} / \mathrm{H}]$ measurements based on such spectra are presented in Hill et al. (2011). The comparison between the equivalent widths (EWs) of CaT lines in the LR08 spectra and the $[\mathrm{Fe} / \mathrm{H}]$ abundances, obtained from the EWs of individual FeI lines, yields a linear relationship that extends up to $[\mathrm{Fe} / \mathrm{H}] \sim+0.5$ dex and is fully compatible with the analogous relations obtained by Cole et al. (2004) and Warren et al. (2009). The details of the new calibration will be discussed in a separate paper. We emphasize here that it has been constructed precisely for bulge giants and particularly tested for RC stars.

In order to convert EWs into metallicities, it is necessary to calculate the so-called reduced EWs, a parametrization that removes the effects of temperature and gravity on the EWs, defined as

$W^{\prime}=\Sigma E W+\beta\left(K-K_{\mathrm{RC}}\right)$,

where $K_{\mathrm{RC}}$ is the mean $K_{\mathrm{s}}$ magnitude of the RC. In our case, $K_{\mathrm{RC}}$ was obtained independently for the BRC and FRC using the photometric catalogue from the VVV survey (Saito et al. 2012) and extinction maps from Gonzalez et al. (2012). The quantity $\Sigma E W$ is the sum of the EWs of the three calcium lines. The measurement of the EWs of CaT lines is rather difficult for stars of solar and super-solar metallicity, due to the presence of many small molecular lines that contaminate the pseudo-continuum. In the case of IMACS spectra the task is further complicated by the fact that the dispersion axis goes across four different chips of the mosaic, with gaps between them. Due to this and ti the lower $\mathrm{S} / \mathrm{N}$ of the IMACS spectra with respect to the GIRAFFE ones used to derive the calibration, the metallicity derived from IMACS spectra are not very accurate. In particular, for four stars in common between the IMACS and GIRAFFE samples, the derived metallicities both show an offset (of $\sim 0.2$ dex) with a mild trend with metallicity. Rather than relying on just four stars to measure and correct for this systematics, we prefer to analyze here only the metallicities for the IMACS targets, without combining them with the GIRAFFE ones.

One might argue that we are ignoring the highest quality data (GIRAFFE sample) and keeping only the lower quality ones (IMACS sample). This choice is due to the larger number of IMACS targets and is justified by the assumption that the impact of the systematics in the measurements of EWs in IMACS would be small if metallicities are considered only on a relative scale. The IMACS metallicities will be labeled as instrumental, hereafter, to emphasize that we have indications that they might not be accurate on an absolute scale. Moreover, these CaT metallicities are used uniquely to check whether the bright and faint clumps have consistent or (slightly) different metallicity distributions.

Figure 8 shows the metallicity histogram for the target stars in the BRC (red) and the FRC (blue). As already found by De Propris et al. (2011), the two samples do not show any difference in their mean metallicity. However, if we select only BRC stars with $V_{\mathrm{R}}<-50 \mathrm{~km} \mathrm{~s}^{-1}$ and FRC stars with $V_{\mathrm{R}}>$ $+50 \mathrm{~km} \mathrm{~s}^{-1}$, as already done for the PMs, then a difference of $\sim 0.11$ dex is found between the mean $[\mathrm{Fe} / \mathrm{H}]$ of the two samples. A small difference between the near and the far overdensities of bulge stars at $(l, b)=(0,-6)$ is consistent with the presence of a gradient along the minor axis, as observed by Zoccali et al. (2008), Johnson et al. (2011), and Gonzalez et al. (2011). Indeed, the line of sight crosses the near and the far overdensities at different distances from the Galactic center. In order to estimate the expected difference in metallicity, the distance to each overdensity was determined following McWilliam \& Zoccali (2010), who found a $\Delta Z \sim 200 \mathrm{pc}$ in their distance to the Galactic plane. Assuming a $0.6 \mathrm{dex} / \mathrm{kpc}$ gradient, a difference of 0.12 dex in metallicity would be expected between stars in the two RCs. Clearly, this comparison is very preliminary because the RV cut is arbitrary and the gradient quoted in the literature is based on samples of stars without line-of-sight distance information, i.e., summing up stars in the near and far overdensities in an unknown proportion. In summary, there is evidence in the literature of the presence of a metallicity gradient along the bulge minor axis. While the value of this gradient is quite uncertain, we found here that adopting an RV cut, that minimizes cross-contamination, is possible to measure a metallicity gradient consistent with that reported by Zoccali et al. (2008).

Finally, another interesting piece of evidence is shown in Fig. 9. The metallicity of the BRC (red) and FRC (blue) stars is shown here as a function of RV. A glance at this plot shows some segregation of the FRC stars on the right half of the plane, while the BRC ones are preferentially on the left side. The additional evidence appearing here is that this segregation is stronger for metal-poor stars $([\mathrm{Fe} / \mathrm{H}]<0)$, shown in the lower left-hand panel, than it is for the metal-rich ones $([\mathrm{Fe} / \mathrm{H}]>0)$, shown 

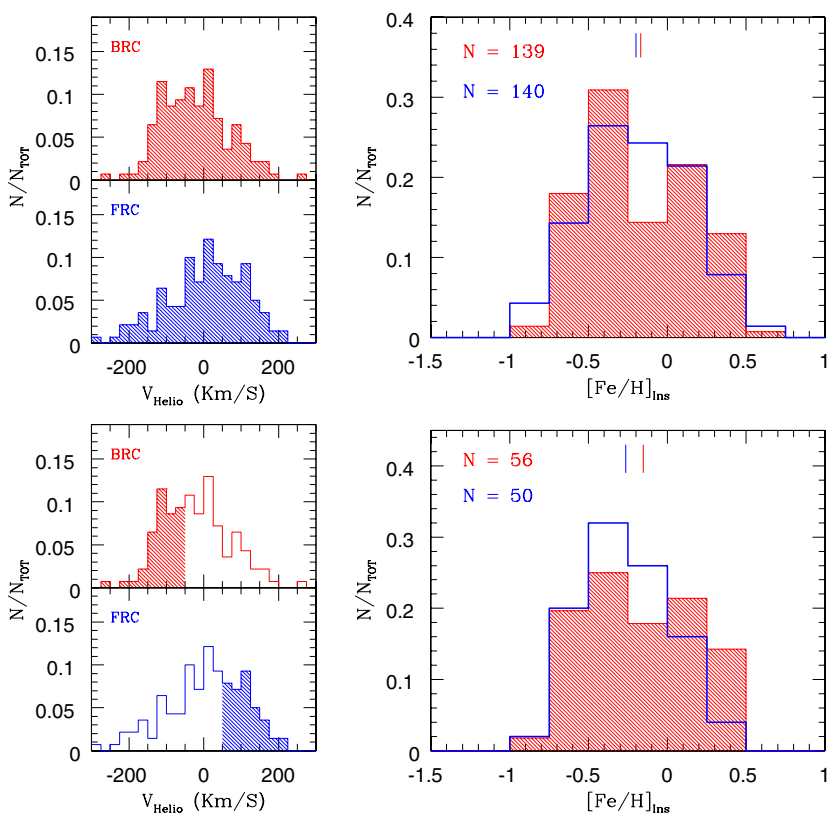

Fig. 8. Instrumental metallicity distribution for IMACS data. When all the BRC and FRC in whole RV regime (upper left panels) are selected, the $[\mathrm{Fe} / \mathrm{H}]_{\text {Ins }}$ distribution plotted in upper right panel does not show a statistical difference. Nevertheless, when a selection is done in the same way as Fig. 6 (lower left panels), a shift in the $[\mathrm{Fe} / \mathrm{H}]_{\text {Ins }}$ distribution is observed in the lower right panel. The star number is also indicated in each case, following the key color adopted in this paper and the corresponding selection shown in the left panels.

in the lower right-hand panel. Surprisingly, this would argue in favor of the metal-poor stars being preferentially in the more elongated orbits, defining the X-shape, while the most metalrich ones are preferentially found on axisymmetric orbits, which are centered at zero RV and with lower velocity dispersion.

Ness et al. (2012) found that metal-rich RC stars have a smaller velocity dispersion than metal-poor ones, which is consistent with what we see in Fig. 8. They also found that the magnitude (distance) separation between the two RCs is stronger for the metal-rich stars. The latter evidence cannot be checked here because our initial target selection excluded stars in between the two RCs. Our results that the RV asymmetry is stronger for the metal-poor half of the sample, however, suggest that the magnitude splitting should be there, perhaps even stronger, for these stars.

\section{Conclusions}

We have analysed a sample of 454 bulge stars equally distributed between the BRC and the FRC of a bulge field at $(l, b)=(0,-6)$. The two RCs are used here as tracers of the near and far overdensities of the X-shaped bulge, which is crossed by the line of sight at these coordinates. We obtained RVs and PMs for all the stars and CaT metallicities for a subsample of 352 stars.

We also measured the PM of NGC 6558, finding an observed mean PM of $\left(\mu_{1} \cos (b), \mu_{b}\right)=(0.30 \pm 0.14,-0.43 \pm 0.13)$, with a dispersion of $\left(\sigma_{l} \cos (b), \sigma_{b}\right)=(1.8,1.7) \mathrm{mas} / \mathrm{yr}$. This is the first PM measurement for NGC 6558.

An excess of bulge stars in elongated orbits $\left(\left|V_{\mathrm{R}}\right|>\right.$ $50 \mathrm{~km} \mathrm{~s}^{-1}$ ) has been found in both RCs. The near overdensity contains preferentially stars with negative RVs, while the far one contains preferentially stars with positive RVs. The 3D velocities of these stars are qualitatively consistent with the predictions
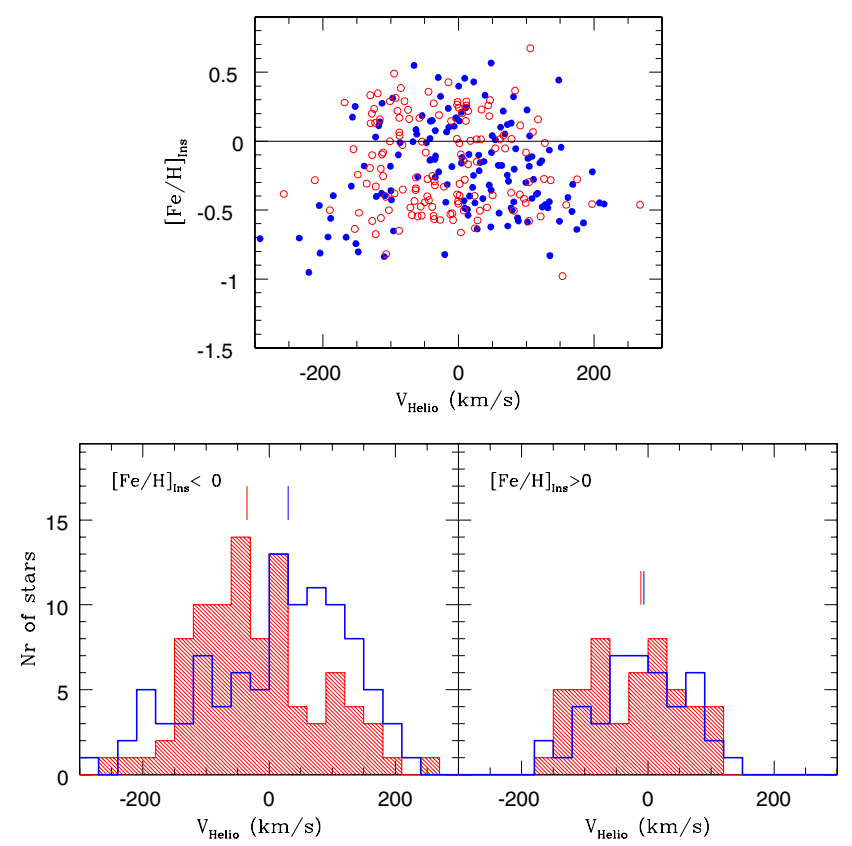

Fig. 9. $[\mathrm{Fe} / \mathrm{H}]$ versus $\mathrm{RV}$ for $\mathrm{BRC}$ and $\mathrm{FRC}$ samples observed with IMACS. The histograms show an interesting difference when the sample is divided into metal-poor and metal-rich.

of $N$-body models by Debattista et al. (2005) with a strong $\mathrm{X}$-shaped bulge.

Interestingly, not all the stars in the two RCs are in elongated orbits. Roughly half of the stars in both the BRC and the FRC share the same 3D velocity, with mean RVs and PMs centered at zero. They seem to be stars in axisymmetric orbits that are somehow coexisting with those in elongated orbits. Whether the two kinematic groups share the same origin, i.e., whether they both belong to the same Galactic component, is impossible to say with the present data. Nevertheless, it is interesting to note that current models predict that the presence of a bar should clean up the inner region of a Galaxy, i.e., no stars in axisymmetric orbits should be left there, regardless of their origin.

Metallicity measurements provide further evidence for the stars in elongated orbits: those with the largest difference in RV and PMs are preferentially the ones with sub-solar metallicities, while the more metal-rich ones in both the near and far overdensity share the same 3D motions, with RV and PMs centered at zero and a smaller RV dispersion. This finding, if confirmed, would be at odds with the suggestion by Babusiaux et al. (2010) that metal-poor bulge stars have spheroid-like kinematics, while the metal-rich ones have a significant vertex deviation, typical of stars in bar-like orbits.

Clearly, all the evidence presented here is based on a relatively small number of stars and needs to be confirmed with larger samples, such as the ESO-Gaia Public Spectroscopic Survey (PIs: Gilmore \& Randich), the ARGOS survey (PI: Freeman), ESO Large Program 187.B-0909 (PI: Zoccali), and the APOGEE survey (PI: Majewski). Indeed, the present pilot study demonstrates the amount of information that can be acquired by combining spectra with multiepoch photometry for red clump stars for which distances are known. For example, with the ESO Large Program we expect to soon be able to use this kind of data for significantly larger samples of stars along many different bulge lines of sight. As a result, important constraints will be set to state-of-the-art dynamical models, with the 
ultimate aim of establishing the relative roles of the processes that contributed to make the Milky Way bulge as we see it today.

Acknowledgements. We thank Santino Cassisi for calculating the expected fraction of red giant branch contamination in the red clump from his models. S.V. and M.Z. acknowledge support by Proyecto Fondecyt Regular 1110393. While working on this paper, M.Z. enjoyed a sabbatical year at the INAF Osservatorio Astronomico di Bologna and the European Southern Observatory in Garching. Both institutions are warmly thanked for their kind hospitality. A fellowship from the John Simon Guggenheim Memorial Foundation has partly financed this stay. This work is supported by the BASAL Center for Astrophysics and Associated Technologies PFB-06, the FONDAP Center for Astrophysics 15010003, Proyecto Anillo ACT-86, and the Chilean Ministry for the Economy, Development, and Tourism's Programa Iniciativa Científica Milenio through grant P07-021-F, which was awarded to The Milky Way Millennium Nucleus. M.M. was suppported by the IAC (grants 310394, 301204) and the Education and Science Ministry of Spain (grants AYA2010-16717).

\section{References}

Athanassoula, E. 2003, MNRAS, 341, 1179 Athanassoula, E. 2005, MNRAS, 358, 1477 Athanassoula, E. 2012, MNRAS, 426, L46 Babusiaux, C., \& Gilmore, G. 2005, MNRAS, 358, 1309 Babusiaux, C., Gómez, A., Hill, V., et al. 2010, A\&A, 519, A77 Barbuy, B., Zoccali, M., Ortolani, S., et al. 2007, AJ, 134, 1613 Binney, J., Gerhard, O., Spergel, D. 1997, MNRAS, 288, 365

Bissantz, N., \& Gerhard, O. 2002, MNRAS, 330, 591

Bournaud, F., Elmegreen, B. G., \& Martig, M. 2009, ApJ, 707, L1

Cabrera-Lavers, A., Hammersley, P. L., González-Fernndez, C., et al. 2007, A\&A, 465, 825

Cabrera-Lavers, A., González-Fernández, C., Garzón, F., et al. 2008, A\&A, 491, 781

Carollo, C. M., Scarlata, C., Stiavelli, M., et al. 2007, ApJ, 658, 960 Churchwell, E., Babler, B. L., Meade, M. R., et al. 2009, PASP, 121, 213

Clarkson, W., Sahu, K., Anderson, J., et al. 2008, ApJ, 684, 1110

Clarkson, W. I., Sahu, K. C., Anderson, J., et al. 2011, ApJ, 735, 37

Cole, A. A., Smecker-Hane, T. A., Tolstoy, E., et al. 2004, MNRAS, 347, 367

Combes, F., \& Sanders, R. H. 1981, A\&A, 96, 164

Daddi, E., Bournaud, F., Walter, F., et al. 2010, ApJ, 713, 686

De Propris, R., Rich, R. M., Kunder, A., et al. 2011, ApJ, 732, L32

Debattista, V. P., Carollo, C. M., Mayer, L., et al. 2005, ApJ, 628, 678

Debattista, V. P., Mayer, L., Carollo, C. M., et al. 2006, ApJ, 645, 209

Dehnen, W. 2000, AJ, 119, 800

Dehnen, W., \& Binney, J. J. 1998, MNRAS, 298, 387

Dwek, E., Arendt, R. G., Hauser, M. G., et al. 1995, ApJ, 445, 716

Elmegreen, B. G., Bournaud, F., \& Elmegreen, D. M. 2008, ApJ, 688, 67

Emsellem, E., Cappellari, M., Krajnović, D., et al. 2011, MNRAS, 414, 888

Feltzing, S., \& Johnson, R. A. 2002, A\&A, 385, 67

Förster Schreiber, N. M., Genzel, R., Lehnert, M. D., et al. 2006, ApJ, 645, 1062

Genzel, R., Newman, S., Jones, T., et al. 2011, ApJ, 733, 101

Gonzalez, O. A., Rejkuba, M., Zoccali, M., et al. 2011, A\&A 530, A54

Gonzalez, O. A., Rejkuba, M., Zoccali, M., et al. 2012, A\&A, 543, A13

Hammersley, P. L., Garzón, F., Mahoney, T. J., et al. 2000, MNRAS, 317, L45

Hanuschik, R. W. 2003, A\&A, 407, 1157

Harris, W. E. 1996, AJ, 112, 1487

Hill, V., Lecureur, A., Gómez, A., et al. 2011, A\&A, 534, A80
Howard, C. D., Rich, R. M., Reitzel, D. B., et al. 2008, ApJ, 688, 1060 Howard, C. D., Rich, R. M., Clarkson, W., et al. 2009, ApJ, 702, L153 Immeli, A., Samland, M., Gerhard, O., \& Westera, P. 2004, A\&A, 413, 547 Johnson, D. R. H., \& Soderblom, D. R. 1987, AJ, 93, 864

Johnson, C. I., Rich, R. M., Fulbright, J. P., et al. 2011, ApJ, 732, 108

Kent, S. M. 1992, AJ, ApJ, 387, 181

Kuijken, K., \& Rich, R. M. 2002, AJ, 124, 2054

Kunder, A., Koch, A., Rich, R. M., et al. 2012, AJ, 143, 57

López-Corredoira, M., Cabrera-Lavers, A., Mahoney, T. J., et al. 2007, AJ, 133 , 154

Martinez-Valpuesta, I., \& Gerhard, O. 2011, ApJ, 734, L20

Martinez-Valpuesta, I., Shlosman, I., \& Heller, C. 2006, ApJ, 637, 214

McWilliam, A., \& Zoccali, M. 2010, ApJ, 724, 1491

Minniti, D. 1996, ApJ, 459, 175

Nataf, D. M., Udalski, A., Gould, A., et al. 2010, ApJ, 721, L28

Ness, M., Freeman, K., Athanassoula, E., et al. 2012, ApJ, 756, 22

Ortolani, S., Renzini, A., Gilmozzi, R. et al. 1995, Nature, 377, 701

Patsis, P. A., Skokos, Ch., \& Athanassoula, E. 2002, MNRAS, 337, 578

Pryor, C., \& Meylan, G. 1993, ASPC, 50, 357

Rangwala, N., \& Williams, T. B. 2009, ApJ, 702, 414

Rangwala, N., Williams, T. B., \& Stanek, K. Z. 2009, ApJ, 691, 1387

Rattenbury, N. J., Mao, S., Sumi, T., et al. 2007, MNRAS, 378, 1064

Rich, R. M. 1988, AJ, 95, 828

Rich, R. M. 1990, ApJ, 362, 604

Rich, R. M., \& Terndrup, D. 1997, ASPC, 127, 129

Roeser, S., Demleitner, M., \& Schilbach, E. 2010, AJ, 139, 2440

Robin, A. C., Reyle, C., Derriere, S., et al. 2003, A\&A, 409, 523

Robin, A. C., Marshall, D. J., Schultheis, M., et al. 2012, A\&A, 538, A106

Sadler, E. M., Rich, R. M., \& Terndrup, D. M. 1996, AJ, 112, 171

Saha, K., Tseng, Y., \& Taam, R. E. 2010, ApJ, 721, 1878

Saha, K., Martinez-Valpuesta, I., \& Gerhard, O. 2012, MNRAS, 421, 333

Saito, R., Zoccali, M., McWilliam, A., et al. 2011, AJ, 142, 76

Saito, R. K., Hempel, M., Minniti, D., et al. 2012, A\&A, 537, A107

Sellwood, J. A., \& Valluri, M. 1997, MNRAS, 287, 124

Schönrich, R., Binney, J., \& Dehnen, W. 2010, MNRAS, 403, 1829

Scott D. W. 1985, The Annals of Statistics, 13, 1024

Shen, J., Rich, R. M., Kormendy, J., et al. 2010, ApJ, 720, L72

Skrutskie, M. F., Cutri, R. M., Stiening, R., et al. 2006, AJ, 131, 1163

Soto, M., Rich, R. M., \& Kuijken, K. 2007, ApJ, 665, L31

Spaenhauer, A., Jones, B. F., \& Whitford, A. E. 1992, AJ, 103, 297

Stanek, K. Z., Mateo, M., Udalski, A., et al. 1994, ApJ, 429, L73

Stetson, P. B. 1994, PASP, 106, 250

Stetson, P. B., \& Harris, W. E. 1988, AJ, 96, 909

Sumi, T., Wu, X., Udalski, A., et al. 2004, MNRAS, 348, 1439

Szymanski, M. K., Udalski, A., Soszynski, I., et al. 2011, Acta Astron., 61, 83

Tacconi, L. J., Genzel, R., Neri, R., et al. 2010, Nature, 463, 781

Taylor, M. B., 2005, in Astronomical Data Analysis Software and Systems, XIV ASP Conf. Ser., eds. P. Shopbell, M. Britton, \& R. Ebert (San Francisco, ASP), 347, 29

Terndrup, D. M., Sadler, E. M., \& Rich, R. M. 1995, AJ, 110, 1774

Toomre, A. 1977, in Evolution of Galaxies and Stellar Populations, eds. B. M. Tinsley, \& R. B. Larson (New Haven: Yale University Observatory), 401

Uttenthaler, S., Schultheis, M., Nataf, D. M., et al. 2012, A\&A, 546, A57

Vieira, K., Casetti-Dinescu, D., Mendez, R., et al. 2007, AJ, 134. 1432

Warren, Steven R., \& Cole, A. A. 2009, MNRAS, 393, 272

Zacharias, N. 2010, AJ, 139, 2184

Zhao, H. S. 1996, MNRAS, 283, 149

Zoccali, M., Renzini, A., Ortolani, S., et al. 2001, AJ, 121, 2638

Zoccali, M., Renzini, A., Ortolani, S., et al. 2003, A\&A, 399, 931

Zoccali, M., Hill, V., Lecureur, A., et al. 2008, A\&A, 486, 177 